\title{
Free energy loss analysis of heterojunction solar cells
}

\author{
Nils Brinkmann*, Gabriel Micard, Yvonne Schiele, Giso Hahn, and Barbara Terheiden
}

Department of Physics, University of Konstanz, P.O. Box X916, 78457 Konstanz, Germany

Keywords loss analysis, simulations, heterojunction solar cells, free energy

* Corresponding author: e-mail nils.brinkmann@uni-konstanz.de, Phone: +49 7531 882088, Fax: +49 7531883895

For the first time we present a free energy loss analysis (FELA) of heterojunction silicon solar cells (HSSC) to study the influence of the intrinsic buffer layer thickness $\left(t_{\text {buffer }}\right)$ on the solar cell efficiency $(\eta)$. The main advantage of the FELA is that the impact of various loss mechanisms can be directly expressed in absolute percentage of $\eta$. Furthermore, it is possible to extract the magnitude of every loss for each region of the solar cell. All quantities required to perform the FELA are obtained by the simulation software AFORS-HET. The FELA yields an optimum efficiency of $21.24 \%$ for $t_{\text {buffer }} \approx 5 \mathrm{~nm}$. The efficiency drop for $t_{\text {buffer }} \leq 5 \mathrm{~nm}$ is ascribed to a lower maximum usable generated power $\Phi_{\mathrm{G}}(22.84 \%$ @ $2 \mathrm{~nm}, 23.98 \% @ 5 \mathrm{~nm}$ ). Lower efficiencies for $t_{\text {buffer }} \geq 5 \mathrm{~nm}$ are attributed to the increased transport loss of holes in the intrinsic buffer layer (0.05\%@2 nm,0.65\%@8 nm). The $\eta$ values yielded by the FELA are in agreement with the ones calculated by AFORS-HET, demonstrating the applicability of the FELA to the HSSC concept. Therewith, we demonstrate that the FELA can be employed to obtain a deeper understanding of the HSSC concept.
1 Motivation Recently, the heterojunction solar cell concept, which utilizes stacked films composed of an intrinsic amorphous silicon (a-Si:H) passivation and a doped a-Si:H emitter layer, has been attracted much attention [1]. Its key advantages are the high conversion efficiency potential $(24.7 \%)$ [2] and the fact that the whole solar cell production process can be carried out at economical and energy efficient temperatures below $200{ }^{\circ} \mathrm{C}$ [3].

Quite a few simulation studies aiming at finding the optimal structure of the heterojunction solar cell using AFORS-HET or 1-DAMPS have already been published (e.g. $[4,5])$. All of these studies investigate the influence of certain cell parameters on the IV-curve and thus on the output parameters of the solar cell. However, for heterojunction solar cells a loss analysis that is the absolute quantification of the various loss mechanisms has not been published so far to our knowledge. So, in this Letter, we apply for the first time the free energy loss analysis (FELA) [6] to obtain a more profound understanding of the heterojunction solar cell concept. The FELA is a very recent type of loss analysis in which the maximum power available to perform work in the external circuit (the input rate of free energy) as well as all electrical loss mechanisms (recombi- nation and transport) can be quantified in $\mathrm{mW} / \mathrm{cm}^{2}$. Alternatively, these quantities can be expressed directly in absolute efficiency units (\%) just by normalizing them by the total power density of the AM1.5G spectrum. Furthermore, it is possible to assign the magnitude of every loss to each region of the solar cell. Both properties turn the FELA into a powerful tool for analyzing heterojunction solar cells. In this study, we focus on the influence of the intrinsic buffer layer thickness upon the efficiency of the solar cell in terms of free energy dissipation rates.

\section{Theory}

2.1 Free energy loss analysis The FELA was originally introduced by Brendel et al. [6]. Its starting point is that the electrical power provided by a solar cell is free from entropy. Therefore, it is possible to express this power as a rate of free energy $\left(\mathrm{mW} / \mathrm{cm}^{2}\right)$. The maximum usable power generated by the solar cell can be expressed in one dimension as

$$
\Phi_{\mathrm{G}}=q \int_{0}^{t} G \cdot\left(\Psi_{\mathrm{fn}}-\Psi_{\mathrm{fp}}\right) \cdot \mathrm{d} z
$$


in which $G$ is the generation rate, $\Psi_{\mathrm{fn}}$ and $\Psi_{\mathrm{fp}}$ are the quasiFermi levels for electrons and holes (in V), $t$ represents the thickness of the solar cell and $q$ being the elementary charge. In the same manner the recombination losses in the bulk can be calculated by

$$
\Phi_{R_{\mathrm{v}}}=q \int_{0}^{t} R_{\mathrm{v}} \cdot\left(\Psi_{\mathrm{fn}}-\Psi_{\mathrm{fp}}\right) \cdot \mathrm{d} z,
$$

in which $R_{\mathrm{v}}$ is the total recombination rate that can be further decomposed into different recombination mechanism contributions (Auger, Shockley-Read-Hall (SRH) or radiative).

The recombination losses at each electrode interface can be expressed by

$$
\Phi_{R_{\mathrm{s}}}=J_{\mathrm{MIN}-\mathrm{C}} \cdot\left(\Psi_{\mathrm{fn}}-\Psi_{\mathrm{fp}}\right),
$$

in which $J_{\text {MIN-C }}$ is the current density of minority carriers at the surface.

Finally, the transport losses for electrons are determined by

$$
\Phi_{\mathrm{te}}=\int_{0}^{t} J_{\mathrm{e}}^{2} /\left(q \cdot n \cdot \mu_{\mathrm{e}}\right) \cdot \mathrm{d} z,
$$

in which $J_{\mathrm{e}}$ is the electron current density, $n$ the electron density and $\mu_{\mathrm{e}}$ the electron mobility. An analogous expression can also be derived for holes, $\Phi_{\text {th }}$.

In this way all losses become directly comparable. Since all the volume losses are calculated as integrals over solar cell thickness, it is possible to extract the magnitude of every loss path for each region of the solar cell.

If the FELA is performed at maximum power point, the efficiency can be derived according to

$$
\eta_{\mathrm{FELA}}=\left(\Phi_{G}-\Phi_{R_{\mathrm{v}}}-\Phi_{R_{\mathrm{s}}}-\Phi_{\mathrm{te}}-\Phi_{\mathrm{th}}\right) / \Phi_{\mathrm{AM} 1.5 \mathrm{~g}},
$$

in which $\Phi_{\mathrm{AM} 1.5 \mathrm{~g}}$ is the total incoming power.

2.2 Simulation of heterojunction solar cells The heterojunction solar cells are simulated using AFORSHET, which is a device simulator program for modeling multi-layer heterojunction solar cells in one dimension [7]. A detailed description of AFORS-HET is contained in [8]. We study a non-textured heterojunction solar cell featuring an n-type crystalline silicon base $(200 \mu \mathrm{m}$, doping concentration $N_{\mathrm{d}}=1.5 \times 10^{16} \mathrm{~cm}^{-3}$ ), a p-type a-Si:H front emitter $\left(10 \mathrm{~nm}, N_{\mathrm{a}}=5 \times 10^{19} \mathrm{~cm}^{-3}\right.$, total defect density $\left.N_{\mathrm{tr}}=1.5 \times 10^{18} \mathrm{~cm}^{-3}\right)$ including an intrinsic buffer layer $\left(2-8 \mathrm{~nm}, N_{\mathrm{tr}}=4.7 \times 10^{16} \mathrm{~cm}^{-3}\right)$ and an $\mathrm{n}^{+}$-type a-Si:H $\left(15 \mathrm{~nm}, N_{\mathrm{d}}=7 \times 10^{19} \mathrm{~cm}^{-3}, N_{\text {tr }}=7 \times 10^{19} \mathrm{~cm}^{-3}\right)$ back surface field (BSF). All other input parameters needed for the simulations in this work are chosen according to [9]. In this study we also take into account that interface passivation depends on the thickness of the intrinsic a-Si:H buffer layer [10]. Hence, surface recombination velocity $S_{\text {eff }}$ of FZ-silicon wafers passivated by intrinsic a-Si:H is experi- mentally determined in dependence on the a-Si:H layer thickness. This dependence is empirically parameterized as power law $S_{\text {eff }} \propto t_{\text {buffer }}^{\gamma}$ in which $\gamma=-4$ gave the best fit. Assuming a capture cross-section $\sigma$ independent of $t_{\text {buffer }}$ yields $N_{\mathrm{s}} \propto S_{\text {eff }} \propto t_{\text {buffer }}{ }^{-4}$, and we parameterize $N_{\mathrm{s}}$ as $N_{\mathrm{s}}=N_{\text {ref }} \times\left(t_{\text {buffer }} / t_{\text {ref }}\right)^{-4}$ scaled with $t_{\text {ref }}=5 \mathrm{~nm}$ and $N_{\text {ref }}=5 \times 10^{10} \mathrm{~cm}^{-2}$ according to [11]. $N_{\mathrm{s}}$ therefore ranges from $6.4 \times 10^{12} \mathrm{~cm}^{-2} @ t_{\text {buffer }}=2 \mathrm{~nm}$ to $2.1 \times 10^{9} \mathrm{~cm}^{-2} @$ $t_{\text {buffer }}=8 \mathrm{~nm}$.

To model the interface between the a-Si:H buffer layer and the c-Si absorber, a thin $(1 \mathrm{~nm})$ layer is added, which has a c-Si bandgap with two Gaussian distributed dangling bond defects centered at midgap $\quad$ width $=0.18 \mathrm{eV}, \quad$ correlation $\quad$ energy $=0.15 \mathrm{eV}$, $\left.\sigma^{\text {uncharged }}=10^{-17} \mathrm{~cm}^{2}, \sigma^{\text {charged }}=10^{-18} \mathrm{~cm}^{2}\right)[9,11]$.

AFORS-HET is used not only to calculate efficiency but also to extract all parameters required to perform the FELA $\left(G(z), R_{\mathrm{v}}(z), J_{\mathrm{e}, \mathrm{h}}(z), n(z), h(z), \mu_{\mathrm{e}, \mathrm{h}}(z), \Psi_{\mathrm{fn}, \mathrm{fp}}(z) \mathrm{cf}\right.$. Eq. (1)-(4)). Note, that it is necessary to extract all parameters at maximum power point if the efficiencies obtained by the FELA and AFORS-HET are supposed to be compared.

3 Results and discussion Considering that the energy absorbed per photon equals the bandgap energy $q \times\left(\Psi_{\mathrm{c}}-\Psi_{\mathrm{v}}\right)$, one can derive a quantity $\Phi_{\mathrm{opt}}$ analogously to Eq. (1) as

$$
\Phi_{\mathrm{opt}}=q \int_{0}^{t} G \cdot\left(\Psi_{\mathrm{c}}-\Psi_{\mathrm{v}}\right) \cdot \mathrm{d} z .
$$

$\Phi_{\text {opt }}$ is the ultimate efficiency of a solar cell. For the stack system simulated in this study, it reaches a value of about $43 \%$ and slightly increases with the thickness of the intrinsic a-Si:H buffer layer (Fig. 1).

Unfortunately, almost half of this energy is not electrically usable because it contributes to the entropy increase of the solar cell. Strictly speaking this energy is not lost but cannot be delivered to the device plugged to the solar cell.

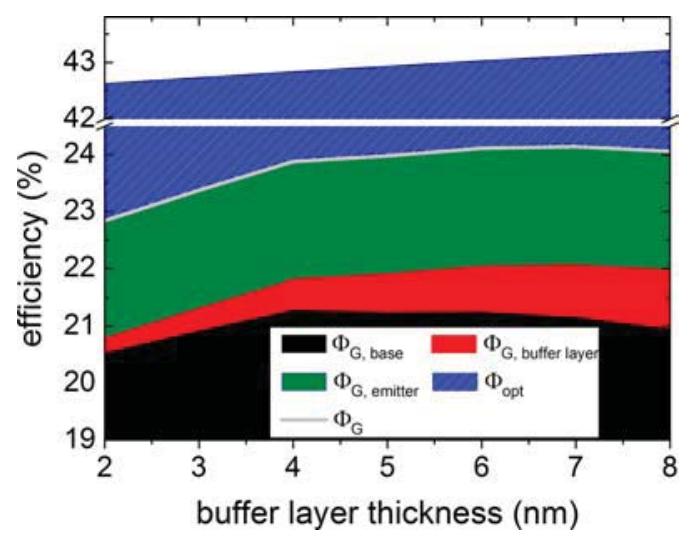

Figure 1 Ultimate efficiency $\Phi_{\mathrm{opt}}$ and maximum usable generated efficiency $\Phi_{\mathrm{G}}$ separated into base, buffer layer and emitter components dependent on buffer layer thickness. 
The maximum usable generated power $\Phi_{\mathrm{G}}$ of the simulated solar cell structure (free of entropy) is depicted in Fig. 1 wherein it is separated into base, buffer layer and emitter component.

$\Phi_{\mathrm{G}}$ increases globally with buffer layer thickness from $22.8 \%(2 \mathrm{~nm})$ to $24.1 \%(8 \mathrm{~nm})$ as a result of a continuously rising $\Phi_{\text {opt }}(42.6-43.2 \%)$. However, $\Phi_{\mathrm{G}}$ features a steeper increase for thicknesses below $4 \mathrm{~nm}(23.9 \%$ at $4 \mathrm{~nm}$, cf. Fig. 1), which can therefore be attributed to a smaller QF1 splitting at lower thicknesses of the intrinsic buffer layer. For $t_{\text {buffer }} \leq 4 \mathrm{~nm}$, this smaller QF1 splitting can be mainly attributed to a lower minority carrier density in the base but also in the thinner intrinsic layer, both induced by a higher recombination rate at the interface.

Whereas $\Phi_{\mathrm{G}}$ stays rather constant within the doped a-Si:H emitter layer, generated power within the intrinsic a-Si:H buffer layer increases with its thickness (cf. Eq. (1) and Fig. 1). This yields a diminishment of $\Phi_{\mathrm{G}}$ within the base with increasing buffer layer thickness above $4 \mathrm{~nm}$, since the additional usable power generated within the buffer layer is no longer available for generation within the base.

However, due to recombination and transport losses only part of this maximum generated free energy is utilizable for the solar cell.

$\Phi_{\mathrm{G}}$ including all recombination losses $\Phi_{R}$ (SRH and Auger), except the negligible recombination losses in the intrinsic buffer layer, are depicted in Fig. 2.

With thinner buffer layers, i.e. with growing $N_{\mathrm{s}}$, the recombination losses at the interface clearly increase from $0.03 \%$ at $8 \mathrm{~nm}$ to $0.64 \%$ at $2 \mathrm{~nm}$. But surprisingly at the same time recombination losses within the base decrease with the result that the sum of both recombination losses is more or less independent of buffer layer thickness (1.27 $\pm 0.07 \%$, cf. Fig. 2).

If the interface is strongly recombinative (small buffer layer thickness), it causes carriers driving from the base towards the interface to recombine. This in turn reduces the carrier density in the base and thus the recombination rate independently of the carrier lifetime. On the other

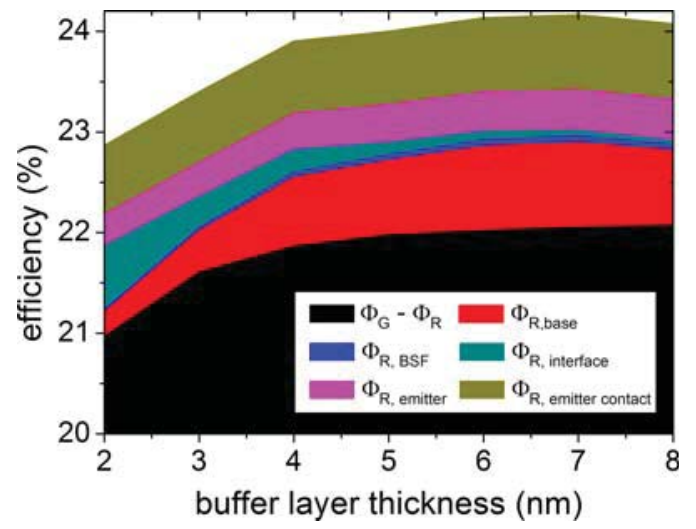

Figure $2 \Phi_{\mathrm{G}}-\Phi_{\mathrm{R}}$ and all significant recombination losses dependent on buffer layer thickness.

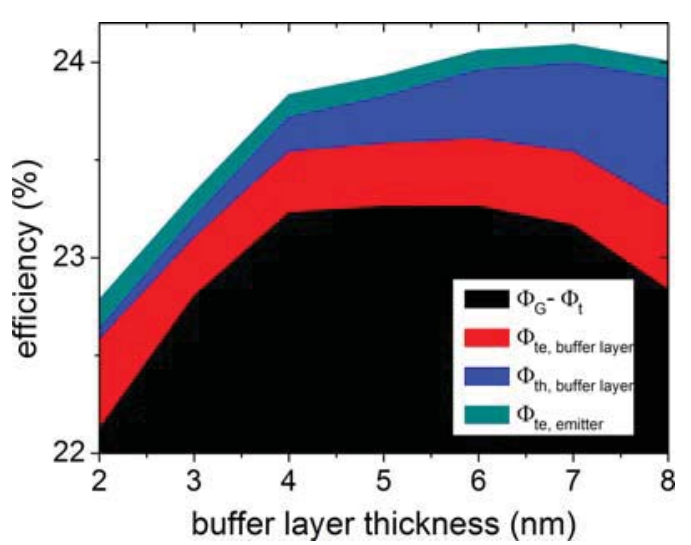

Figure $3 \Phi_{\mathrm{G}}-\Phi_{\mathrm{t}}$ and all significant transport losses dependent on buffer layer thickness.

hand, if the surface is not strongly recombinative (large buffer layer thickness), very few carriers are driven from the base to the interface. This results in an increase of the carrier density in the base and therewith of the recombination rate.

Recombination losses in the doped a-Si:H emitter $(0.31-0.41 \%)$ and the a-Si:H back surface field (BSF) $(0.02-0.06 \%)$ only slightly increase with buffer layer thickness, which can be attributed to a more efficient QF1 splitting.

Whereas recombination loss at the BSF contact is completely negligible $\left(<1 \times 10^{-4} \%\right), \Phi_{R_{\mathrm{S}}}$ at the emitter contact accounts for $0.69 \%(2 \mathrm{~nm})$ to $0.75 \%(8 \mathrm{~nm})$.

In the final analysis, the overall recombination losses increase only slightly with ascending buffer layer thickness and account for an efficiency loss of $1.9 \%(2 \mathrm{~nm})$ to $2.1 \%$ $(8 \mathrm{~nm})$.

The maximum generated power including the major transport losses for holes and electrons $\left(\Phi_{\mathrm{t}}=\Phi_{\mathrm{th}}+\Phi_{\mathrm{te}}\right)$ in the intrinsic buffer layer and the doped emitter layer are depicted in Fig. 3. The not shown transport losses (e.g. in the BSF and the base) sum up to an average of $0.07 \pm 0.003 \%$ and are therefore neglected in the following discussion.

$\Phi_{\mathrm{t}}$ is mainly caused by transportation of electrons and holes through the intrinsic buffer layer (Fig. 3). Whereas transport loss of electrons stays almost constant $(0.36 \%)$, the transport loss of holes increases with increasing buffer layer thickness from $0.05 \%(2 \mathrm{~nm})$ to $0.65 \%(8 \mathrm{~nm})$. The origin of this is still under discussion.

The maximum generated power $\Phi_{\mathrm{G}}$ as well as all losses are summarized in Fig. 4. Again, despite a strongly increasing $N_{\mathrm{s}}$ value with decreasing intrinsic buffer layer thickness resulting in an increase of its recombination activity, it is clearly observable that the sum of all recombination losses remains almost constant for all simulated structures.

The only reason for the decrease in efficiency with buffer layer thickness is the separation of QFl, which depends indirectly on the interface defect density. This result 


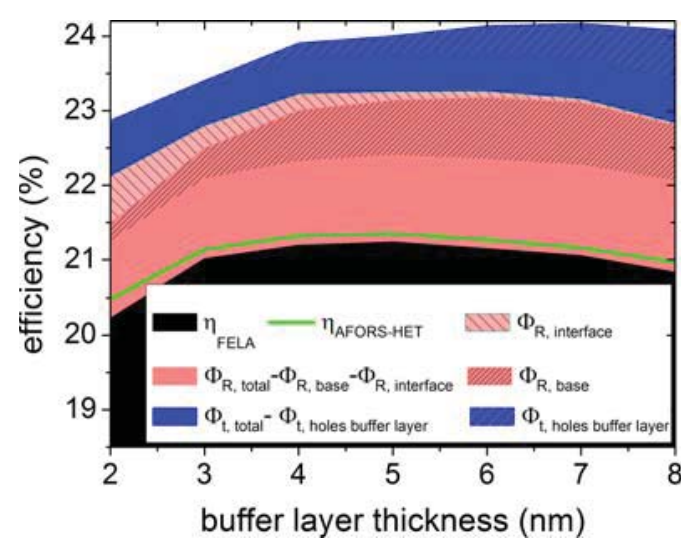

Figure 4 Efficiencies derived by FELA and AFORS-HET as well as all recombination and transport losses in dependence of buffer layer thickness. Major contributors to the losses are indicated.

is in accordance with the open-circuit voltage $\left(V_{\mathrm{oc}}\right)$ deduced from AFORS-HET which increases with intrinsic buffer layer thickness from $690 \mathrm{mV}(2 \mathrm{~nm})$ to $740 \mathrm{mV}$ $(8 \mathrm{~nm})$.

Despite the increasing $\Phi_{\mathrm{G}}$ and the almost constant recombination losses with increasing buffer layer thickness, there is a clear decrease of efficiency for buffer layer thicknesses above $5 \mathrm{~nm}$ (from $21.23 \%$ at $5 \mathrm{~nm}$ to $20.83 \%$ at $8 \mathrm{~nm}$ ). The decreasing efficiency for thicker buffer layers can be attributed to an increase of $\Phi_{\text {th }}$ in the intrinsic layer. This is again in accordance with the results deduced from AFORS-HET since the fill factor is reduced by around $4 \%$ abs from 2 to $8 \mathrm{~nm}$ buffer layer thickness.

The efficiency yielded by the FELA is in agreement $(0.67 \pm 0.15 \%$ rel $)$ with the calculated efficiency of AFORSHET (Fig. 4) proving the results deduced from the FELA to be consistent, since a small discrepancy between $\eta_{\text {FELA }}$ and the simulated efficiency $\eta_{\text {AFORS-HET }}$ can be considered as a cross-check of the consistency of the whole approach. This discrepancy results from the discretization of the problem and the multiplicity of the quantities involved in the FELA calculation and is therefore unavoidable.

4 Conclusion For the first time we have applied the free energy loss analysis (FELA) to the heterojunction solar cell concept.

We have shown that the efficiencies computed out of the FELA and efficiencies simulated by AFORS-HET are in agreement, demonstrating the consistency and reliability of the FELA.

In this work, we have studied the influence of the thickness of the intrinsic buffer layer $\left(t_{\text {buffer }}\right)$ on efficiency losses.

The FELA yields an optimum efficiency for $t_{\text {buffer }}$ $\approx 5 \mathrm{~nm}$. Lower efficiencies for $t_{\text {buffer }} \leq 5 \mathrm{~nm}$ are attributed to (a) a lower maximum usable generated power $\Phi_{\mathrm{G}}$, which increases globally with $t_{\text {buffer }}$ as a result of a continuously raising ultimate efficiency $\Phi_{\text {opt }}$, and (b) to a higher recom- bination activity at the interface, which induces a reduction of $\Phi_{\mathrm{G}}$ due to a lower minority carrier density in the base, yielding a smaller QFl splitting.

Lower efficiencies for $t_{\text {buffer }} \geq 5 \mathrm{~nm}$ are attributed to the increased transport loss of holes in the intrinsic buffer layer (0.05\%@2 nm,0.65\%@8 nm).

Additionally, we have remarked that the sum of base and interface recombination losses is independent of $t_{\text {buffer }}$ $(1.27 \pm 0.07 \%)$ and that the recombination losses in the emitter and at the emitter contact correspond to half of the generated power in the emitter.

In general, recombination losses are always higher than transport losses. However, if one considers the additional lateral transport loss in the transparent conductive oxide (TCO) and the contact fingers, this might be the opposite.

Although the interpretation of FELA results is not trivial, the expression of all losses in absolute percentage of efficiency as well as its possible application to any solar cell concept turns the FELA into a powerful method.

Acknowledgements N. Brinkmann gratefully acknowledges the sponsorship by the scholarship program of the German Federal Environmental Foundation (Deutsche Bundesstiftung Umwelt, DBU).

\section{References}

[1] S. De Wolf, A. Descoeudres, Z. C. Holman, and C. Ballif, Green 2, 7 (2012).

[2] Press Release: http://www.pv-tech.org/news/recombination_ loss_improvements_key_to_panasonic_hit_cell_efficiency_ of_- 24 , date 12.02. 2013 .

[3] S. Taira, Y. Yoshimine, T. Baba, M. Taguchi, H. Kanno, T. Kinoshita, H. Sakata, E. Maruyama, and M. Tanaka, in: Proc. 22nd EUPVSEC, Milan, Italy, 2007, pp. 932-935.

[4] L. Zhao, C. L. Zhou, H. L. Li, H. W. Diao, and W. J. Wang, Sol. Energy Mater. Sol. Cells 92, 673 (2008).

[5] N. Hernandez-Como and A. Morales-Acevedo, Sol. Energy Mater. Sol. Cells 94, 62 (2010).

[6] R. Brendel, S. Dreissigacker, N.-P. Harder, and P. P. Altermatt, Appl. Phys. Lett. 93, 173503 (2008).

[7] R. Stangl, M. Kriegel, and M. Schmidt, in: Proc. 20th EUPVSEC, Barcelona, Spain, 2005, pp. 814-817.

[8] R. Stangl and C. Leendertz, in: Physics and Technology of Amorphous-Crystalline Heterostructure Silicon Solar Cells, edited by W. G. J. H. M. van Sark, L. Korte, and F. Roca, Gerneral Principles of Solar Cell Simulation and Introduction to AFORS-HET (Springer, Berlin-Heidelberg, 2012), chap. 13.

[9] C. Leendertz and R. Stangl, in: Physics and Technology of Amorphous-Crystalline Heterostructure Silicon Solar Cells, edited by W. G. J. H. M. van Sark, L. Korte, and F. Roca, Modelling an a-Si:H/c-Si Solar Cell with AFORS-HET, (Springer, Berlin-Heidelberg, 2012), chap. 14.

[10] H. Plagwitz, Surface passivation of crystalline silicon solar cells by amorphous silicon films, Dissertation, Gottfried Wilhelm Leibniz Universität, Hannover, 2007, p. 137.

[11] C. Leendertz, N. Mingirulli, T. F. Schulze, J. P. Kleider, and B. Rech, Appl. Phys. Lett. 98, 202108 (2011). 